2013, Vol. 1, No. 3

\title{
Relationship between Economic Security and Country Risk Indicators in EU Baltic Sea Region Countries
}

\author{
Jelena Stankevičienè, Tatjana Sviderskè, Algita Miečinskienė
}

\begin{abstract}
A B S T R A C T
Objective: The globalization phenomenon raises new challenges in terms of country risk and economic security for small open economies. The objective of this paper is to evaluate the relationship between economic security and country risk indicators in EU Baltic Sea region countries.

Research Design \& Methods: This paper, after surveying definitions and typologies of risks, proposes the analysis of the relationship between economic security and country risk in EU Baltic Sea region countries based on statistical data from 2012. The results were optimized by implementing MOORA (Multi-Objective Optimization by Ratio analysis) and MULTIMOORA (MOORA plus Full Multiplicative Form) methods.
\end{abstract}

Findings: Findings provide evidence for economic security being dependent on country risk ratios. This result is robust with respect to the applied method of investigation.

Implications \& Recommendations: It is crucial to identify the potential for different types of risks, security indicators as well as methods for risk evaluation and assessment. The key variables of interest include domestic economic variables, macroeconomic policy evaluation, balance of payments stability and social indicators. A general sustainability context (monetary, social and environmental indicators) should be also taken into consideration.

Contribution \& Value Added: Several investigations come to strongly conclusive results, which could be used in creating a new model for country risk assessment and the derived economic security indicators for EU Baltic Sea region countries.

$\begin{array}{ll}\text { Article type: } & \text { research paper } \\ \text { Keywords: } & \text { Economic security; globalization; EU Baltic Sea region; } \\ & \text { MULTIMOORA }\end{array}$

JEL codes:

G11, G14, G23

Published by Centre for Strategic and International Entrepreneurship - Krakow, Poland

\section{Suggested citation:}

Stankevičienè, J., Sviderskè, T., \& Miečinskienė, A. (2013). Relationship between Economic Security and Country Risk Indicators in EU Baltic Sea Region Countries. Entrepreneurial Business and Economics Review, 1(3), 21-33. 


\section{INTRODUCTION}

The notion of economic security is quickly gaining attention and its importance has increased in the last years. Contemporary globalization - economic integration at the global level that is no longer limited to the industrialized countries - accelerated during the 1980s, as programs of economic liberalization spread throughout the developing world. A huge increase in capital flows to developing countries in the early 1990s reinforced positive views of globalization. Financial crises at the turn of the 21st century were caused by international effects and the United States high technology boom and its accompanying stock market bubble. They were a good push in governments' understanding of economic security. The economic crises and their repercussions occurring in an increasingly integrated global economy have spurred renewed interest in economic security and created initiatives to redefine it. This revised definition in turn has encouraged a search for policy prescriptions that will increase economic security in the new environment.

Globalization, after undermining the old definition of economic security, is at the centre of a new definition that emphasizes the risks of unexpected shocks and economic volatility. The new definition must capture the causal consequences of globalization accurately and establish explicit benchmarks for assessing globalization's effects on economic security. Economic security is not a new concern of governments. Earlier, economic instruments have long been part of the governmental strategy, as a mean to influence other states and their policies. Economic security in this traditional view was independence from manipulation by other governments that wielded these instruments.

\section{THEORETHICAL BACKGROUND: DEFINITIONS OF ECONOMIC SECURITY}

Economic security is a topic, which is quite rarely approached by researchers. Very often, the significance of this issue is fully understood only post factum, when the threats to the economic security of a country have had effect (Geršl \& Heřmánek, 2006). Economic history proves that economic security should become the object of a permanent monitoring and management system (Heslop \& Helen, 2009; Hlaváček, 2007).

According to Huber, Rehm, Schlesinger, and Valletta (2010) economic security could be considered as a preparation state of the economy for ensuring decent conditions for living and developing the socio-economic stability and the political-military capability of the society and the country in order to eliminate internal and external threats. Generally, there is no universal definition of the concept of economic security, because of its multilateral and multidimensional features.

After analysing of different scientific articles and different opinions of researchers (Kesternich \& Schnitzer, 2010; Bordo, Meissner, \& Weidenmier, 2009; Busse \& Hefeker, 2006; Finnerty, 2001), it is clear that the concept of economic security is complex and dynamic. Its complexity stems from the multitude of economic, social, financial processes, as well as, from the phenomena of globalisation (Miskiewicz \& Ausloos, 2010; Scheve, Kenneth, \& Slaughter, 2002), seen both as a process and as a phenomenon acting systematically and permanently upon national economies. Its dynamism is caused by the quick pace of the economic processes and phenomena on both national and global level (Reuer \& Leiblein, 2000). 
Economic security should be understood as (Rehm \& Schlesinger, 2013; Quadrini, 2011; Ausloos \& Miskiewicz, 2010; Rehm \& Schlesinger, 2010; Marshall, Maulana, \& Tang, 2009; den Besten, 2007; Estrada, 2000; Meldrum, 2000):

- an essential factor of national security, ensuring resources and the dynamic balance of all other components of this system,

- a dimension of national, regional and global security, which is an aim of every individual, community, country, etc.,

- a priority objective of governments, regional and international organizations working to ensure and guarantee global human security,

- a state of the national economy, seen as a source and basis for eliminating poverty, famine, social and economic inequalities, both between individuals and between regions of a country.

Most of the definitions of economic security provided by researchers from various countries (Ratha, De Prabal, \& Mohapatra, 2011; Schroeder, 2008; Quer, Claver, \& Rienda, 2007) may be classified into three categories:

- definitions that identify economic security with its objectives,

- definitions that identify economic security with a state of the economy, which implies several favourable consequences,

- definitions that consider economic security as an element of production stability.

The country's economic security is determined by three main components: economic security of countries, companies and consumers. The balance of the three is crucial for the security of the whole country's economy. The main objective of the country's economic security consists of ensuring basic conditions for the country's socioeconomic development (Rehm, Hacker, \& Schlesinger, 2012; Osberg \& Sharpe, 2009).

The concept of economic security has a lot of milestones, which should be considered: it lacks the historical primacy and intellectual currency assigned to military security; it suffers from a diffuseness of both potential threats and remedies; and its content resists clear categories of threat.

Further analysis will show how important it is to distinguish the dependence of economic security on country risk indicators, as by this approach, many decisions could be made, evaluating different types of opportunities.

\section{RESEARCH METHODS: MOORA AND MULTIMOORA METHODS}

Multi-Objective Optimization by Ratio Analysis (MOORA) method was introduced by Brauers and Zavadskas (2006). This method was developed (Brauers \& Zavadskas, 2010) and became MULTIMOORA (MOORA plus the full multiplicative form). These methods have been applied in different studies (Brauers, Ginevičius, Zavadskas, \& Antuchevičienè, 2007; Brauers \& Ginevičius, 2009; Brauers \& Zavadskas 2009; Brauers \& Ginevičius, 2010; Baležentis A., Baležentis, T., \& Valkauskas, 2010; Brauers, Ginevičius, \& Podvezko, 2010).

According to Brauers and Zavadskas (2006), MOORA goes for a ratio system in which each response of an alternative on an objective is compared to a denominator, which is representative for all alternatives concerning that objective.

MOORA method begins with the matrix $X$ where its elements $x_{i j}$ denote $j$-th alternative of $i$-th objective $(i=1,2, \ldots, n$ and $j=1,2, \ldots, m)$. In our case we have $m=8$ alternatives (EU 
Baltic Sea region countries) and $n=23$ objectives (indicators). MOORA method consists of two parts: the ratio system and the reference point approach.

\section{The Ratio System of MOORA}

The ratio system defines data normalization by comparing alternative of an objective to all values of the objective (1):

$$
x_{i j}^{*}=\frac{x_{i j}}{\sqrt{\sum_{j=1}^{m} x_{i j}^{2}}}
$$

where:

$x_{i j}$ - response of alternative $j$ on objective $i$,

$j=1,2, \ldots, \mathrm{m} ; \mathrm{m}$ - number of alternatives,

$i=1,2, \ldots, \mathrm{n} ; \mathrm{n}$ - number of objectives,

$x^{*} i j-$ a dimensionless number representing the normalized response of alternative $j$ on objective $i$.

These responses of the alternatives on the objectives belong to the interval $[0 ; 1]$. These indicators are added (if desirable value is maximal) or subtracted (if desirable value is minimal) and summary index of a country derives according to the formula (2):

$$
y_{j}^{*}=\sum_{i=1}^{i=g} x_{i j}^{*}-\sum_{i=g+1}^{i=n} x_{i j}^{*}
$$

where:

$i=1,2, \ldots, g$ as the objectives to be maximized,

$i=g+1, \mathrm{~g}+2, \ldots, \mathrm{n}$ as the objectives to be minimized,

$x_{j}^{*}$-the normalized assessment of alternative $j$ with respect to all objectives.

\section{The Reference Point of MOORA}

This reference point theory starts from the already normalized ratios as defined in the MOORA method. The $j$-th coordinate of the reference point can be described as $r_{j}=\max x_{i j}^{*}$ in maximization case. Every coordinate of this vector represents maximum or minimum of certain objective. Then every element of normalized responses matrix is recalculated and final rank is given according to the deviation from the reference point and the Min-Max Metric of Tchebycheff (3):

$$
\min _{i}\left(\max _{j}\left|r_{j}-x_{i j}^{*}\right|\right)
$$

\section{The Full Multiplicative Form of Multiple Objectives and MULTIMOORA}

Brauers and Zavadskas (2010) proposed updated MOORA with the Full Multiplicative Form method embodying maximization as well as minimization of purely multiplicative utility function. Overall utility of the j-th alternative can be expressed as dimensionless number (4): 


$$
U_{j}^{\prime}=\frac{A_{j}}{B_{j}}
$$

where:

$$
\begin{aligned}
& A_{j}=\prod_{g=1}^{i} x_{g i}, \\
& j=1,2, \ldots, m, \\
& m \text { - number of alternatives, } \\
& i \text { - number of objectives to be maximized, } \\
& B_{j}-\prod_{k=i+1}^{n} x_{k j}, \\
& \text { n-i - number of objectives to be minimized, } \\
& U_{j}^{\prime}-\text { utility of alternative } \mathrm{j} \text { with objectives to be maximized and objectives to } \\
& \quad \text { be minimized. }
\end{aligned}
$$

Thus MULTIMOORA summarizes MOORA (which includes Ratio System and Reference point) and the Full Multiplicative Form.

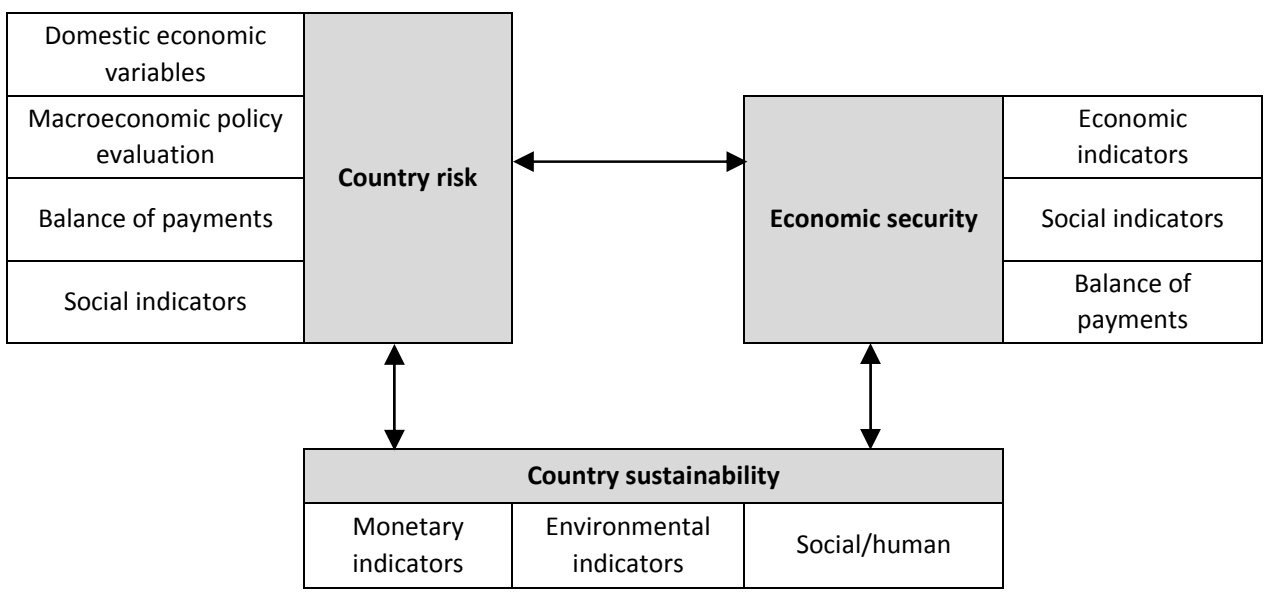

Figure 1. Interdependence between ratios Source: own study.

\section{ANALYSIS OF COUNTRY RISK AND ECONOMIC SECURITY VARIABLES}

The main goal of this study is to determine the relationship between country risk, economic sustainability and economic security (Figure 1). In this article relationship between country risk and economic security ratios will be analysed. There is an assumption, proposed by the authors, that all three variables are interrelated with each other in one or another direction/dependence. This hypothesis has been already proved by several scientific researches (Stankevičienè, Sviderskè, \& Miečinskienè, 2014; Stankevičienè \& Sviderskè, 2012). 
After consolidating different types of variables', different groups of country risk and economic security indicators were created (Table 1 and Table 2).

Table 1. Grouping of indicators for country risk evaluation

\begin{tabular}{|c|c|c|c|}
\hline $\begin{array}{c}\text { Domestic economic } \\
\text { variables }\end{array}$ & $\begin{array}{c}\text { Macroeconomic policy } \\
\text { evaluation }\end{array}$ & Balance of payments & Social indicators \\
\hline $\begin{array}{c}\text { Gross domestic } \\
\text { investment (\% of GDP) }\end{array}$ & $\begin{array}{c}\text { Inflation (End of Year } \\
\text { Change \%) }\end{array}$ & $\begin{array}{c}\text { The current account } \\
\text { balance (\% of GDP - 3 } \\
\text { year average) }\end{array}$ & $\begin{array}{c}\text { Unemployment Rate } \\
\text { (\% of Labour Force) }\end{array}$ \\
\hline GDP (PPP - billion USD) & $\begin{array}{c}\text { Real effective exchange } \\
\text { rate }\end{array}$ & $\begin{array}{c}\text { Balance of trade } \\
\text { (million EUR) }\end{array}$ & $\begin{array}{c}\text { Natural population } \\
\text { change }\end{array}$ \\
\hline $\begin{array}{c}\text { Private consumption (\% } \\
\text { of GDP) }\end{array}$ & $\begin{array}{c}\text { Current taxes on } \\
\text { income, wealth, etc. (\% } \\
\text { of GDP) }\end{array}$ & $\begin{array}{c}\text { Exports of goods and } \\
\text { services (\% of GDP) }\end{array}$ & $\begin{array}{c}\text { Employment (annual } \\
\text { averages) }\end{array}$ \\
\hline
\end{tabular}

Source: created by authors.

Table 2. Grouping of indicators for country's economic security evaluation

\begin{tabular}{|c|c|c|}
\hline Economic indicators & Social indicators & Balance of payments \\
\hline $\begin{array}{ll}\text { o } & \text { Total intramural R\&D } \\
& \text { expenditure (GERD) } \\
& \text { (EUR/inhab.). } \\
\text { o } & \text { High-tech exports (\% of } \\
& \text { exports). } \\
\text { o } & \text { Gross fixed capital formation } \\
& \text { (investments) MEUR. } \\
\text { - } & \text { General government } \\
& \text { deficit/surplus (\% of GDP). } \\
\text { o } & \text { General government gross } \\
& \text { debt (MEUR). }\end{array}$ & 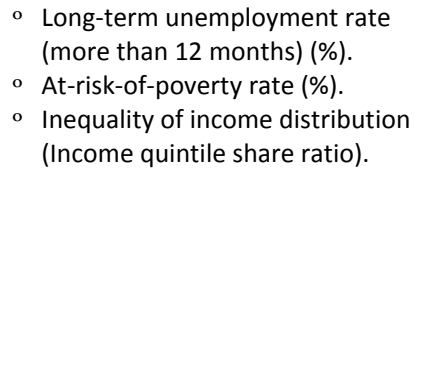 & $\begin{array}{ll} & \text { Balance of international } \\
\text { trade in goods (\% of GDP). } \\
\text { o } \text { Market integration by type } \\
\text { of trade activities (\%). } \\
\text { o Share of import from EU in } \\
\text { total imports (\%). }\end{array}$ \\
\hline
\end{tabular}

Source: created by authors.

For country risk, four main groups of variables were distinguished - domestic economic variables, macroeconomic policy evaluation, balance of payments and social indicators. Each group includes a set of three indicators, which mostly describe country risk.

For economic security, three main groups of variables were distinguished economic indicators, social and balance of payments. Each group includes a set of indicators, which describe countries' economic security (Saisana \& Saltelli, 2010; Saaty, 2010).

\section{RESULTS AND DISCUSSION}

All data for analysis was received from European Statistics Database (Eurostat) and International Monetary Fund for EU Baltic Sea region countries. The data therefore covers eight EU Baltic Sea region countries, year 2012 (latest available data) and 23 structural indicators, 184 observations in total. The indicators used for calculations are presented in Table 3 and Table 4. 
Table 3. Country risk indicators for EU Baltic Sea region countries for 2012

\begin{tabular}{|l|r|r|r|r|r|r|}
\hline \multirow{2}{*}{ Countries } & \multicolumn{3}{|c|}{ Domestic economic variables } & \multicolumn{2}{c|}{ Macroeconomic policy evaluation } \\
\cline { 2 - 6 } & $\begin{array}{c}\text { Gross domestic } \\
\text { investment (\% of GDP) }\end{array}$ & $\begin{array}{c}\text { GDP (PPP - } \\
\text { USD, billions) }\end{array}$ & $\begin{array}{c}\text { Private } \\
\text { consumption (\% of } \\
\text { GDP) }\end{array}$ & $\begin{array}{c}\text { Inflation (End } \\
\text { of Year Change } \\
\text { \%) }\end{array}$ & $\begin{array}{c}\text { Real effective } \\
\text { exchange rate }\end{array}$ & $\begin{array}{c}\text { Current taxes } \\
\text { on income, } \\
\text { wealth, etc. } \\
\text { (\% of GDP) }\end{array}$ \\
\hline Denmark & 17.32 & 210.15 & 49.50 & 1.96 & 96.20 & 30.40 \\
Estonia & 27.63 & 29.09 & 51.80 & 3.76 & 111.30 & 7.00 \\
Finland & 18.74 & 197.48 & 56.30 & 2.45 & 15.90 \\
Germany & 17.22 & 3197.07 & 57.60 & 2.04 & 93.70 & 12.10 \\
Latvia & 25.89 & 37.27 & 62.10 & 1.60 & 116.10 & 7.70 \\
Lithuania & 17.10 & 65.01 & 64.20 & 2.93 & 109.30 & 4.90 \\
Poland & 21.08 & 800.93 & 61.20 & 2.40 & 100.60 & 7.20 \\
Sweden & 18.54 & 392.96 & 48.20 & 1.04 & 100.80 & 18.30 \\
\hline
\end{tabular}

\begin{tabular}{|c|c|c|c|c|c|c|}
\hline \multirow[b]{2}{*}{ Countries } & \multicolumn{3}{|c|}{ Balance of payments } & \multicolumn{3}{|c|}{ Social indicators } \\
\hline & $\begin{array}{c}\text { The current account } \\
\text { balance (\% of GDP - } 3 \\
\text { year average) }\end{array}$ & \begin{tabular}{|c|}
$\begin{array}{c}\text { Balance of } \\
\text { trade (million } \\
\text { EUR) }\end{array}$ \\
\end{tabular} & $\begin{array}{c}\text { Exports of goods } \\
\text { and services (\% of } \\
\text { GDP) }\end{array}$ & \begin{tabular}{|c|} 
Unemployment \\
Rate (\% of \\
Labour Force) \\
\end{tabular} & $\begin{array}{c}\text { Natural } \\
\text { population } \\
\text { change }\end{array}$ & $\begin{array}{c}\text { Employment } \\
\text { (annual } \\
\text { averages) } \\
\end{array}$ \\
\hline Denmark & 5.60 & 585.40 & 54.50 & 7.55 & 1.00 & 2688.60 \\
\hline Estonia & 1.30 & -203.50 & 92.50 & 9.77 & -1.10 & 624.40 \\
\hline Finland & -0.60 & -527.90 & 39.70 & 7.68 & 1.40 & 2483.20 \\
\hline Germany & 6.50 & 16097.60 & 51.60 & 5.46 & -2.30 & 40062.10 \\
\hline Latvia & -0.30 & -135.90 & 61.10 & 14.94 & -4.50 & 885.60 \\
\hline Lithuania & -1.40 & 40.30 & 84.20 & 13.25 & -3.50 & 1278.50 \\
\hline Poland & -4.50 & -788.20 & 46.20 & 10.35 & 0.00 & 15590.70 \\
\hline Sweden & 7.00 & 459.90 & 48.70 & 7.90 & 2.20 & 4657.10 \\
\hline
\end{tabular}

Source: own study.

Table 4. Economic security indicators for EU Baltic Sea region countries for 2012

\begin{tabular}{|c|c|c|c|c|c|}
\hline \multirow[b]{2}{*}{ Countries } & \multicolumn{5}{|c|}{ Economic indicators } \\
\hline & $\begin{array}{c}\text { Total intramural R\&D } \\
\text { expenditure (GERD) } \\
\text { (EUR/inhabitant) }\end{array}$ & \begin{tabular}{|} 
High-tech \\
exports (\% of \\
exports)
\end{tabular} & $\begin{array}{c}\text { Gross fixed capital } \\
\text { formation } \\
\text { (investments) } \\
\text { MEUR }\end{array}$ & $\begin{array}{c}\text { General } \\
\text { government } \\
\text { deficit/surplus } \\
\text { (\% of GDP) }\end{array}$ & $\begin{array}{c}\text { General government gross } \\
\text { debt (MEUR) }\end{array}$ \\
\hline Denmark & 1311.50 & 9.50 & 42638.50 & -4.10 & 110980.20 \\
\hline Estonia & 284.90 & 14.10 & 4392.00 & -0.20 & 1712.10 \\
\hline Finland & 1264.90 & 7.30 & 37868.00 & -1.80 & 103145.00 \\
\hline Germany & 951.00 & 13.90 & 470550.00 & 0.10 & 2160192.50 \\
\hline Latvia & 71.70 & 6.30 & 5072.80 & -1.30 & 9038.00 \\
\hline Lithuania & 98.90 & 5.80 & 5483.60 & -3.20 & 13333.10 \\
\hline Poland & 89.00 & 5.90 & 72981.60 & -3.90 & 217691.00 \\
\hline Sweden & 1464.90 & 12.90 & 77454.90 & -0.20 & 158000.30 \\
\hline
\end{tabular}

\begin{tabular}{|l|r|r|r|r|r|r|}
\hline \multirow{1}{*}{ Countries } & \multicolumn{3}{|c|}{ Social } & \multicolumn{2}{c|}{ Balance of payments } \\
\cline { 2 - 7 } & $\begin{array}{c}\text { Long-term } \\
\text { unployment rate } \\
\text { (more than 12 } \\
\text { months) (\%) }\end{array}$ & $\begin{array}{c}\text { At-risk-of- } \\
\text { poverty rate } \\
\text { (\%) }\end{array}$ & $\begin{array}{c}\text { Inequality of } \\
\text { income distribution } \\
\text { Income quintile } \\
\text { share ratio) }\end{array}$ & $\begin{array}{c}\text { Balance of } \\
\text { international } \\
\text { trade in goods } \\
\text { (\% of GDP) }\end{array}$ & $\begin{array}{c}\text { Market } \\
\text { integration by } \\
\text { type of trade } \\
\text { activities (\%) }\end{array}$ & $\begin{array}{c}\text { Share of } \\
\text { import from } \\
\text { EU in total } \\
\text { imports (\%) }\end{array}$ \\
\hline Denmark & 2.10 & 13.10 & 4.50 & 2.20 & 32.40 & 70.70 \\
Estonia & 5.50 & 17.50 & 5.40 & -4.30 & 76.10 & 80.00 \\
Finland & 1.60 & 13.20 & 3.70 & 0.10 & 29.20 & 62.80 \\
Germany & 2.50 & 16.10 & 4.30 & 6.70 & 41.00 & 63.50 \\
Latvia & 7.80 & 19.20 & 6.50 & -9.80 & 49.50 & 78.20 \\
Lithuania & 6.60 & 18.60 & 5.30 & -3.20 & 71.80 & 56.80 \\
Poland & 4.10 & 17.10 & 4.90 & -1.40 & 39.20 & 67.20 \\
Sweden & 1.50 & 14.10 & 3.70 & 2.10 & 32.00 & 67.10 \\
\hline
\end{tabular}

Source: own study. 
Table 5. Country risk indicators for EU Baltic Sea region countries, normalized by MOORA

\begin{tabular}{|c|c|c|c|c|c|c|}
\hline \multirow[b]{2}{*}{ Countries } & \multicolumn{3}{|c|}{ Domestic economic variables } & \multicolumn{3}{|c|}{ Macroeconomic policy evaluation } \\
\hline & $\begin{array}{c}\text { Gross domestic } \\
\text { investment (\% of } \\
\text { GDP) }\end{array}$ & $\begin{array}{l}\text { GDP (PPP - US } \\
\text { \$,billions) }\end{array}$ & $\begin{array}{c}\text { Private consumption } \\
\text { (\% of GDP) }\end{array}$ & $\begin{array}{l}\text { Inflation (End of } \\
\text { Year Change \%) }\end{array}$ & $\begin{array}{l}\text { Real effective } \\
\text { exchange rate }\end{array}$ & \begin{tabular}{|c}
$\begin{array}{c}\text { Current taxes on } \\
\text { income, wealth, etc. } \\
(\% \text { of GDP) }\end{array}$ \\
\end{tabular} \\
\hline Denmark & 0.29 & 0.06 & 0.31 & 0.27 & 0.33 & 0.71 \\
\hline Estonia & 0.47 & 0.01 & 0.32 & 0.52 & 0.38 & 0.16 \\
\hline Finland & 0.32 & 0.06 & 0.35 & 0.48 & 0.33 & 0.37 \\
\hline Germany & 0.29 & 0.96 & 0.36 & 0.28 & 0.32 & 0.28 \\
\hline Latvia & 0.44 & 0.01 & 0.39 & 0.22 & 0.40 & 0.18 \\
\hline Lithuania & 0.29 & 0.02 & 0.40 & 0.41 & 0.37 & 0.11 \\
\hline Poland & 0.36 & 0.24 & 0.38 & 0.33 & 0.34 & 0.17 \\
\hline Sweden & 0.32 & 0.12 & 0.30 & 0.14 & 0.35 & 0.43 \\
\hline \multirow[b]{2}{*}{ Countries } & \multicolumn{3}{|c|}{ Balance of payments } & \multicolumn{3}{|c|}{ Social indicators } \\
\hline & $\begin{array}{c}\text { The current } \\
\text { account balance } \\
\text { (\% of GDP - } 3 \text { year } \\
\text { average) }\end{array}$ & $\begin{array}{c}\text { Balance of trade } \\
\text { (mil.EUR) }\end{array}$ & $\begin{array}{c}\text { Exports of goods and } \\
\text { services (\% of GDP) }\end{array}$ & $\begin{array}{l}\text { Unemployment } \\
\text { Rate (\% of } \\
\text { Labour Force) }\end{array}$ & $\begin{array}{l}\text { Natural } \\
\text { population } \\
\text { change }\end{array}$ & $\begin{array}{c}\text { Employment } \\
\text { (annual averages) }\end{array}$ \\
\hline Denmark & 0.46 & 0.04 & 0.31 & 0.27 & 0.15 & 0.06 \\
\hline Estonia & 0.11 & -0.01 & 0.52 & 0.34 & -0.16 & 0.01 \\
\hline Finland & -0.05 & -0.03 & 0.23 & 0.27 & 0.20 & 0.06 \\
\hline Germany & 0.54 & 1.00 & 0.29 & 0.19 & -0.34 & 0.92 \\
\hline Latvia & -0.02 & -0.01 & 0.35 & 0.53 & -0.66 & 0.02 \\
\hline Lithuania & -0.12 & 0.00 & 0.48 & 0.47 & -0.51 & 0.03 \\
\hline Poland & -0.37 & -0.05 & 0.26 & 0.36 & 0.00 & 0.36 \\
\hline Sweden & 0.58 & 0.03 & 0.28 & 0.28 & 0.32 & 0.11 \\
\hline
\end{tabular}

Source: own study.

Table 6. Economic security indicators for EU Baltic Sea region countries, normalized by MOORA

\begin{tabular}{|c|c|c|c|c|c|c|}
\hline \multirow[b]{2}{*}{ Countries } & \multicolumn{6}{|c|}{ Economic indicators } \\
\hline & $\begin{array}{c}\text { Total intramural } \\
\text { R\&D expenditure } \\
\text { (GERD) } \\
\text { (EUR/inhab.) }\end{array}$ & $\begin{array}{l}\text { High-tech } \\
\text { exports (\% of } \\
\text { exports) }\end{array}$ & $\begin{array}{c}\text { Gross fixed capital } \\
\text { formation } \\
\text { (investments) MEUR }\end{array}$ & $\begin{array}{c}\text { General } \\
\text { government } \\
\text { deficit/surplus } \\
\text { (\% of GDP) }\end{array}$ & \multicolumn{2}{|c|}{$\begin{array}{l}\text { General government gross debt } \\
\text { (MEUR) }\end{array}$} \\
\hline Denmark & 0.52 & 0.33 & 0.09 & -0.60 & \multicolumn{2}{|l|}{0.05} \\
\hline Estonia & 0.11 & 0.50 & 0.01 & -0.03 & \multicolumn{2}{|l|}{0.00} \\
\hline Finland & 0.50 & 0.26 & 0.08 & -0.26 & \multicolumn{2}{|l|}{0.05} \\
\hline Germany & 0.37 & 0.49 & 0.97 & 0.01 & \multicolumn{2}{|l|}{0.99} \\
\hline Latvia & 0.03 & 0.22 & 0.01 & -0.19 & \multicolumn{2}{|l|}{0.00} \\
\hline Lithuania & 0.04 & 0.20 & 0.01 & -0.47 & \multicolumn{2}{|l|}{0.01} \\
\hline Poland & 0.03 & 0.21 & 0.15 & -0.57 & \multicolumn{2}{|l|}{0.10} \\
\hline Sweden & 0.58 & 0.45 & 0.16 & -0.03 & \multicolumn{2}{|c|}{0.07} \\
\hline & \multicolumn{3}{|c|}{ Social } & \multicolumn{3}{|c|}{ Balance of payments } \\
\hline Countries & $\begin{array}{c}\text { Long-term } \\
\text { unemployment } \\
\text { rate (more than } 12 \\
\text { months) (\%) }\end{array}$ & $\begin{array}{c}\text { At-risk-of- } \\
\text { poverty rate (\%) }\end{array}$ & $\begin{array}{l}\text { Inequality of income } \\
\text { distribution (Income } \\
\text { quintile share ratio) }\end{array}$ & $\begin{array}{c}\text { Balance of } \\
\text { international } \\
\text { trade in goods } \\
\text { (\% of GDP) }\end{array}$ & \begin{tabular}{|c|} 
Market \\
integration by \\
type of trade \\
activities (\%)
\end{tabular} & $\begin{array}{l}\text { Share of import } \\
\text { from EU in total } \\
\text { imports (\%) }\end{array}$ \\
\hline Denmark & 0.16 & 0.28 & 0.33 & 0.16 & 0.23 & 0.36 \\
\hline Estonia & 0.43 & 0.38 & 0.39 & -0.32 & 0.54 & 0.41 \\
\hline Finland & 0.12 & 0.29 & 0.27 & 0.01 & 0.21 & 0.32 \\
\hline Germany & 0.19 & 0.35 & 0.31 & 0.50 & 0.29 & 0.33 \\
\hline Latvia & 0.60 & 0.42 & 0.47 & -0.73 & 0.35 & 0.40 \\
\hline Lithuania & 0.51 & 0.40 & 0.38 & -0.24 & 0.51 & 0.29 \\
\hline Poland & 0.32 & 0.37 & 0.36 & -0.10 & 0.28 & 0.35 \\
\hline Sweden & 0.12 & 0.31 & 0.27 & 0.16 & 0.23 & 0.35 \\
\hline
\end{tabular}

Source: own study.

The initial data was normalized according to formula (1) for Ratio System of MOORA, and then formula (2) was used for obtaining ranks of the Ratio System of MOORA. Formula (3) was applied for the ratios obtained according to formula (1) for Ratio System of MOORA. At the end, initial data was computed according to formula (4), 
providing ranks of the Full Multiplicative Form. Final ranks were obtained through the dominance theory (Brauers, 2004). The results are presented in Table 5 for country risk indicators and in Table 6 for economic security indicators ${ }^{1}$.

After data is normalized, the correlation analysis (Mirkin, 2011; Miskiewicz, 2012) could be presented in order to understand the relationship between each variable for each country risk and economic security group (Table 7).

Table 7. Correlation matrix between country risk and economic security indicators for EU Baltic Sea region countries

\begin{tabular}{|c|c|c|c|c|c|c|c|c|c|c|c|c|}
\hline \multirow{2}{*}{\multicolumn{2}{|c|}{ Indicators }} & \multirow[b]{2}{*}{ 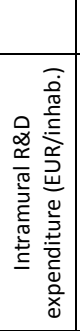 } & \multirow[b]{2}{*}{ 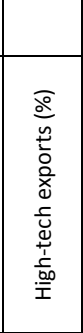 } & \multicolumn{9}{|c|}{ Economic security } \\
\hline & & & & 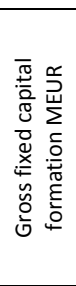 & 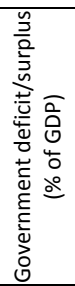 & 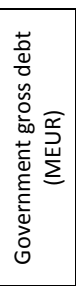 & 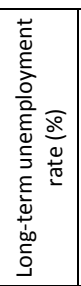 & 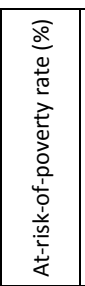 & 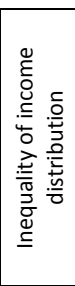 & 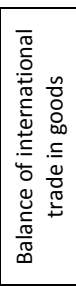 & 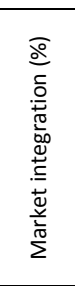 & 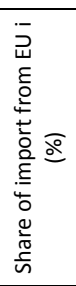 \\
\hline \multirow{12}{*}{ 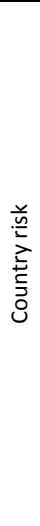 } & $\begin{array}{l}\text { Gross domestic investment (\% } \\
\text { of GDP) }\end{array}$ & -0.558 & 0.083 & -0.391 & 0.314 & -0.358 & 0.604 & 0.538 & 0.675 & -0.744 & 0.491 & 0.863 \\
\hline & GDP (PPP - US \$,billions) & 0.177 & 0.438 & 0.992 & 0.359 & 0.989 & -0.330 & -0.052 & -0.289 & 0.684 & -0.237 & -0.293 \\
\hline & Private consumption (\% of GDP) & -0.755 & \begin{tabular}{|l|l|}
-0.699 \\
\end{tabular} & 0.009 & -0.283 & 0.059 & 0.660 & 0.722 & 0.549 & -0.440 & 0.332 & -0.316 \\
\hline & Inflation (End of Year Change \%) & -0.295 & \begin{tabular}{|l|l|}
-0.069 \\
\end{tabular} & -0.241 & -0.073 & -0.191 & 0.164 & 0.110 & 0.059 & -0.179 & 0.514 & -0.031 \\
\hline & \begin{tabular}{|l|} 
Real effective exchange rate \\
\end{tabular} & -0.744 & -0.230 & -0.556 & 0.104 & -0.511 & 0.915 & 0.811 & 0.869 & -0.909 & 0.733 & 0.507 \\
\hline & $\begin{array}{l}\text { Current taxes on income, } \\
\text { wealth, etc. (\% of GDP) }\end{array}$ & 0.832 & 0.220 & 0.039 & -0.216 & -0.003 & -0.712 & -0.858 & -0.552 & 0.524 & -0.670 & 0.001 \\
\hline & $\begin{array}{l}\text { The current account balance (\% } \\
\text { of GDP - } 3 \text { year average) }\end{array}$ & 0.742 & 0.766 & 0.491 & 0.482 & 0.461 & -0.548 & -0.515 & -0.455 & 0.624 & -0.316 & 0.046 \\
\hline & Balance of trade (mil.EUR) & 0.211 & 0.519 & 0.979 & 0.458 & 0.990 & -0.260 & -0.028 & -0.220 & 0.640 & -0.126 & -0.244 \\
\hline & $\begin{array}{l}\text { Exports of goods and services (\% } \\
\text { of GDP) }\end{array}$ & -0.568 & 0.156 & -0.311 & 0.116 & -0.255 & 0.671 & 0.614 & 0.584 & -0.464 & 0.968 & 0.272 \\
\hline & $\begin{array}{l}\text { Unemployment Rate (\% of } \\
\text { Labour Force) }\end{array}$ & -0.789 & -0.644 & -0.617 & -0.252 & -0.576 & 0.910 & 0.785 & 0.845 & -0.920 & 0.538 & 0.228 \\
\hline & Natural population change & 0.753 & 0.263 & -0.096 & -0.084 & -0.168 & -0.860 & -0.877 & -0.827 & 0.535 & -0.626 & -0.107 \\
\hline & Employment (annual averages) & 0.104 & 0.367 & 0.966 & 0.281 & 0.960 & -0.306 & -0.013 & -0.263 & 0.656 & -0.243 & -0.294 \\
\hline
\end{tabular}

Source: own study.

As we can see from Table 7, there are both - positive and negative correlations between variables. The relationship between indicators is quite strong, the strongest correlation is between macroeconomic policy evaluation (country risk group) and social indicators (economic security group), as well as between social indicators (country risk group) and social indicators (economic security group). Domestic economic variables and balance of payments for country risk are also correlating with economic, balance of payment and social indicators for economic security. The strongest negative correlation is between real effective exchange rate (country risk ratio) and balance of international trade in goods for economic security, as well as between unemployment rate (social indicator of country risk) and balance of international trade in goods for balance of payments in economic security. If one indicator increases, another one will be decreasing and vice versa. Positive correlation is between GDP in domestic economic variables and

\footnotetext{
${ }^{1}$ All calculations are available from the authors upon request.
} 
balance of trade in balance of payment (country risk) and gross fixed capital formation and general government gross debt (in economic indicators for economic security). As well, strong positive correlation is between exports of goods and services (balance of payments for country risk) and market integration by type of trade activities (balance of payments for economic security). Ratios of country risk such as inflation do not present a strong correlation with all economic security ratios.

\section{CONCLUSIONS}

The system of 23 indicators for eight EU Baltic Sea region countries for country risk and economic security was introduced. It includes four groups for country risk: domestic economic variables (gross domestic investment, GDP, private consumption), macroeconomic policy evaluation (inflation, real effective exchange rate, current taxes on income, wealth, etc.), balance of payments (current account balance, balance of trade, exports of goods and services) and social indicators (unemployment rate, natural population change, employment rate). Economic security is based on three groups of indicators: Economic indicators (Total intramural R\&D expenditure, high-tech exports, gross fixed capital formation, general government deficit/surplus, general government gross debt), social indicators (long-term unemployment rate, at-risk-of-poverty rate, inequality of income distribution) and balance of payments (balance of international trade in goods, market integration by type of trade activities, share of import from EU in total imports).

Both MOORA method and its updated model MULTIMOORA could be perfectly used while evaluating and standardizing country risk and economic security, as a ratio system, reference point and multiplicative form appropriately suit for cases, where there are several alternatives (EU Baltic Sea region countries) and several objectives (indicators, which directly show country risk and economic security).

After implementation of MOORA method for EU Baltic Sea region countries, it could be concluded that the data was correctly normalized, standardized and optimized. The results are as follows: the correlation between country risk and economic security does exist. The strongest negative correlation is between real effective exchange rate (country risk ratio) and balance of international trade in goods for economic security, as well as between unemployment rate (social indicator of country risk) and balance of international trade in goods for balance of payments in economic security. Strong positive correlation is observed between GDP in domestic economic variables and balance of trade in balance of payment (country risk) and gross fixed capital formation and general government gross debt (in economic indicators for economic security). Strong positive correlation can also be noticed between exports of goods and services (balance of payments for country risk) and market integration by type of trade activities (balance of payments for economic security). Such elements of country risk as inflation are not very influencing all economic security ratios (no strong relationship was detected). It was proved that economic security was related to / dependent on country risk ratios.

For future investigations, new methods for country risk assessment and economic security evaluation could be used (for example, utilizing S\&P ratings) and results compared to those received by using MULTIMOORA method. Furthermore, a new 
investigation on interrelationship between economic security and economic sustainability could be introduced, implementing a three-dimensional analysis.

\section{REFERENCES}

Ausloos, M. \& Miskiewicz, J. (2010). Entropy correlation distance method applied to study correlations between the gross domestic product of rich countries. Int. J. Bifurcat. Chaos, 20:381.

Baležentis, A., Baležentis, T., \& Valkauskas, R. (2010). Evaluating Situation of Lithuania in the European Union: Structural Indicators and MULTIMOORA Method. Technological and Economic Development of Economy, 16(4): 578-602.

Bordo, M. D., Meissner, C. M., \& Weidenmier, M. D. (2009). Identifying the effects of an exchange rate depreciation on country risk: evidence from a natural experiment. Journal of International Money and Finance, 28(6): 1022-1044. doi: 10.1016/j.jimonfin.2008.10.004

Brauers, W. K. M. \& Ginevičius, R. (2009). Robustness in Regional Development Studies. The Case of Lithuania. Journal of Business Economics and Management, 10(2): 121-140.

Brauers, W. K. M. \& Ginevičius, R. (2010). The economy of the Belgian regions tested with MULTIMOORA. Journal of Business Economics and Management, 11(2): 173-209.

Brauers, W. K. M., Ginevičius, R., \& Podvezko, V. (2010). Regional development in Lithuania considering multiple objectives by the MOORA method. Technological and Economic Development of Econom, y 16(4): 613-640.

Brauers, W. K. M., Ginevičius, R., Zavadskas E. K., \& Antuchevičienè J. (2007). The European Union in a transition economy. Transformation in Business \& Economics, 6(2): 21-37.

Brauers, W. K. M. \& Zavadskas, E. K. (2006). The MOORA method and its application to privatization in a transition economy. Control and Cybernetics, 35(2): 445-469.

Brauers, W. K. M. \& Zavadskas, E. K. (2010). Project management by MULTIMOORA as an instrument for transition economies. Technological and Economic Development of Economy, 16(1): 5-24.

Brauers, W. K. M., \& Zavadskas, E. K. (2009). Robustness of the multi-objective MOORA method with a test for the facilities sector. Technological and Economic Development of Economy, 15(2): 352-375.

Busse, M. \& Hefeker, C. (2006). Political risk, institutions and foreign direct investment. European Journal of Political Economy, 23(2): 397-415. doi: 10.1016/j.ejpoleco.2006.02.003

den Besten, P. J. (2007). Risk assessment approaches in European countries. Sustainable Management of Sediment Resources, 3: 153-205. doi: 10.1016/S1872-1990(07)80066-3

Estrada, J. (2000). The cost of equity in emerging markets: a downside risk approach. Emerging Markets Quarterly, 4(3): 19-30.

Finnerty, J. D. (2001). Securitizing political risk investment insurance: lessons from past securitizations, in: Moran, T. H. (ed.) International Political Risk Management. Washington: The World Bank, pp. 77-147.

Geršl, A . \& Heřmánek, J. (2006): Financial Stability Indicators: advantages and disadvantages of their use in the assessment of the financial system stability. Czech National Bank Financial Stability Review.

Heslop, H. B. (2009). Economic Security in an Index of Economic Well-being: The CSLS Indicator of Economic Security. Centre for the Study of Living Standards Research Report 2009-12. 
Hlaváček, M. (2007): Financial Stability Analysis in a Developing Economy. Czech Journal of Economics and Finance, 1-2/2007, pp. 2-4.

Huber, G., Rehm, P., Schlesinger, M., \& Valletta, R. (2010). Economic Security at Risk: Findings from the Economic Security Index.

Kesternich, I. \& Schnitzer, M. (2010). Who is afraid of political risk? Multinational firms and their choice of capital structure. Journal of International Economics, 82(2): 208-218. doi: 10.1016/j.jinteco.2010.07.005

Marshall, A., Maulana, T., \& Tang, L. (2009). The estimation and determinants of emerging market country risk and the dynamic conditional correlation GARCH model. International Review of Financial Analysis, 18(5): 250-259. doi: 10.1016/j.irfa.2009.07.004

Meldrum, D. H. (2000). Country risk and foreign direct investment. Business Economics, 35(1): $33-$ 40.

Mirkin, B. (2011). Core Concepts in Data Analysis: Summarization, Correlation and Visualization. Springer.

Miskiewicz, J. (2012). Analysis of Time Series Correlation. The Choice of Distance Metrics and Network Structure. Acta Phys. Pol. A, 121:B-89-B-94.

Miskiewicz, J. \& Ausloos, M. (2010). Has the world economy reached its globalization limit? Physica A, 389:797-806.

Osberg, L. \& Sharpe, A. (2009). New Estimates of the Index of Economic Well-being for Canada and the Provinces, 1981-2008. Centre for the Study of Living Standards Research Report 2009-10.

Quadrini, V. (2011). Financial Frictions in Macroeconomic Fluctuations. Economic Quarterly, Vol. 97(3), pp. 209-254.

Quer, D., Claver, E., \& Rienda, L. (2007). The impact of country risk and cultural distance on entry mode choice: an integrated approach, Cross Cultural Management. An International Journal, 14(1): 74-87. doi: 10.1108/13527600710718859

Ratha, D., De Prabal, K., \& Mohapatra, S. (2011). Shadow sovereign ratings for unrated developing countries. World Development, 39(3): 295-307. doi: 10.1016/j.worlddev.2010.08.006

Rehm, P. \& Schlesinger, M. (2010). Standing on Shaky Ground: Americans' Experiences with Economic Insecurity.

Rehm, P. \& Schlesinger, M. (2013). The Insecure American: Economic Experiences and Policy Attitudes amid the Great Recession. Perspectives on Politics (March).

Rehm, P., Hacker, J. S., \& Schlesinger, M. (2012). Insecure Alliances: Risk, Inequality, and Support for the Welfare State. American Political Science Review, 106(2): 386-406.

Reuer, J. J. \& Leiblein, M. J. (2000). Downside risk implications of multinationality and international joint ventures. Academy of Management Journal, 43(2): 203-214.

Saaty, T. L. (2010). Principia Mathematica Decernendi: Mathematical Principles of Decision Making. Pittsburgh, Pennsylvania: RWS Publications.

Saisana, M. \& Saltelli, A. (2010). Uncertainty and Sensitivity Analysis of the 2010 Environmental Performance Index, EUR 56990, European Commission, JRC-IPSC, Ispra, Italy.

Scheve, K. \& Slaughter, M. J. (2002). Economic Insecurity and the Globalization of Production. National Bureau of Economic Research Working Paper No. 9339.

Schroeder, S. K. (2008). The underpinnings of country risk assessment. Journal of Economic Surveys, 22(3): 498-535. doi: 10.1111/j.1467-6419.2007.00541.x 
Stankevičienè, J. \& Sviderskè, T. (2012). Country risk assessment based on MULTIMOORA. 7th International Scientific Conference "Business and Management 2012" May 10-11, 2012, Vilnius, Lithuania. doi: 10.3846/bm.2012.069.

Stankevičienè, J., Sviderskè, T., \& Miečinskienè, A. (2014). Comparison of Country Risk, Sustainability and Economic Safety Indices. Business: Theory and Practice, 15(1): 1-10. Advance online publication. doi: 10.3846/btp.2014.01.

\section{Authors}

\section{Jelena Stankevičienè}

Professor at the Department of Finance Engineering at Vilnius Gediminas Technical University (Lithuania). Her main research topics include assets and liability management, regulation of financial institution, financial management for value creation, value engineering.

\section{Tatjana Sviderskè}

PhD student at Vilnius Gediminas Technical University in Lithuania. Her research areas are country risk assessment and management.

\section{Algita Miečinskienè}

Associate Professor at the Department of Finance Engineering at Vilnius Gediminas Technical University (Lithuania). Her main research topics pricing, foreign direct investment, greenfield investments, mergers and acquisitions.

\section{Correspondence to:}

Prof. Jelena Stankevičienè, PhD

Vilnius Gediminas Technical University,

Faculty of Business Management, Saulètekio al., 11-0618, LT-01223 Vilnius, Lithuania jelena.stankeviciene@vgtu.It 
